\title{
Compact Dual-Mode Wideband Filter Based on Complementary Split-Ring Resonator
}

\author{
Amir Ebrahimi, Student Member, IEEE, Withawat Withayachumnankul, Said F. Al-Sarawi, Member, IEEE, and \\ Derek Abbott, Fellow, IEEE
}

\begin{abstract}
This letter proposes a microstrip filter based on a dual-mode complementary split-ring resonator (DMCSRR). For the input/output coupling, a dual $C$-shaped feed structure is implemented together with the DMCSRR to acheive a wideband response. The resonator offers a wide fractional bandwidth of $62 \%$ at the central frequency of $2.23 \mathrm{GHz}$ and a very compact structure with a footprint of $0.0625 \lambda_{g} \times 0.18 \lambda_{g}$, where $\lambda_{g}$ is the guided wavelength at the midband frequency. A comprehensive lumped element circuit analysis accompanies the simulation and measurement results.
\end{abstract}

Index Terms-Compact filter, complementary split-ring resonator (CSRR), dual-mode filter, wideband filter.

\section{INTRODUCTION}

I $\mathrm{N}$ the last decade, the introduction of split-ring resonators (SRRs) has provided new opportunities for designing high performance microwave devices [1]. Split-ring resonators and their counterparts, so called complementary split-ring resonators (CSRRs), have been widely utilized in composite left/right handed transmission lines [2], microwave sensors [3], [4] and filters [5]-[10] due to their compactness, high quality factor $(Q)$, and low radiation loss in RF systems [6].

Recently, there has been significant interest in implementing filters on ground plane with CSRRs. The advantages of these complementary structures lie in their compact size, spurious response suppression [7] and wide-band operation [8]. A bandpass filter is implemented by cascading high-pass and low-pass CSRR-based filter sections in [7]. In another work, a highly selective wideband bandpass filter based on two cascaded CSRRbased filter sections is proposed [9]. However, the cascading scheme is not suited for miniaturization of the filter. Another wideband filter based on a CSRR and square loop feeding resonators has been presented with an accurate equivalent circuit model for investigating the effect of different parameters [10]. All of these earlier implementations only exploit single-mode resonators for their operation.

\footnotetext{
Manuscript received July 30, 2013; revised October 24, 2013; accepted November 08, 2013. Date of publication December 11, 2013; date of current version March 07, 2014.

The authors are with the School of Electrical and Electronic Engineering, The University of Adelaide, Adelaide, SA 5005, Australia (e-mail: amir.ebrahimi@adelaide.edu.au).

Color versions of one or more of the figures in this letter are available online at http://ieeexplore.ieee.org.

Digital Object Identifier 10.1109/LMWC.2013.2291869
}

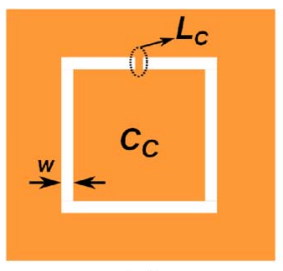

(a)

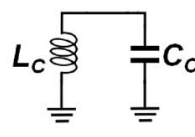

(b)
Fig. 1. Conventional CSRR (a) and its equivalent circuit model (b).

Dual-mode microstrip filters are attractive choices for highly compact microwave systems due to their compact size and fabrication simplicity [11]. This letter demonstrates the design of a wide band compact filter by embracing the concept of DMCSRR. First, the operation principle of dual-mode CSRRs is presented and described by using an equivalent circuit. Next, a $C$-shaped microstrip coupling feed structure [6] is utilized for implementation of high performance dual-mode configuration. Additionally, a comprehensive circuit model is developed for the filter to study its performance as a function of different parameters.

\section{Filter Topology AND Circuit Model}

\section{A. Dual-Mode CSRR}

A single loop conventional CSRR is shown in Fig. 1(a) with its equivalent lumped element circuit model in Fig. 1(b) [12]. In this resonator, $C_{C}$ models the capacitance between the central square plate and the surrounding ground plane separated with a distance $w$, and $L_{C}$ stands for the inductance of the metallic strip connecting the plate to the ground plane. The value of $C_{C}$ depends on the plate circumference and $w$ and $L_{C}$ is a function of $w$ and the strip width. Based on the CSRR in Fig. 1, a dual-mode CSRR can be developed as shown in Fig. 2(a) together with its lumped element circuit model. In the circuit model, $C_{C}$ models the capacitances of the two rectangular plates in the middle surrounded by the ground plane at a distance $w$ from their edges. The inductor $L_{S}$ models the inductances connecting the two capacitive plates to the central parallel inductive path, $L_{P}$, running to the ground plane. Finally, $C_{M}$ represents the mutual capacitance between the two capacitive plates, separated by a width $d$.

Illustrated in Fig. 2(b) are the corresponding even and odd mode equivalent circuits. The inductance $L_{P}$ has no affect on the odd mode operation, whereas the mutual capacitance $C_{M}$ has no affect on the even mode counterpart. From the physical 

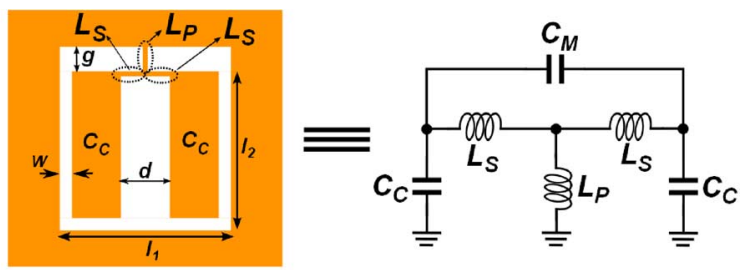

(a)

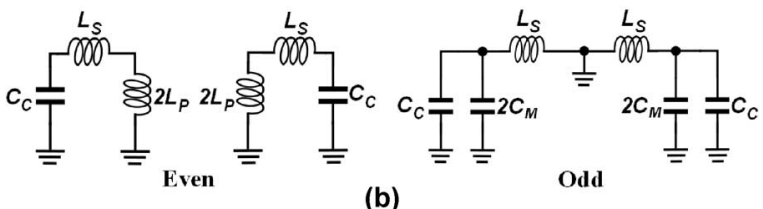

Fig. 2. Dual-mode CSRR: (a) DMCSRR and its equivalent circuit (b) even and odd-mode equivalent circuits.

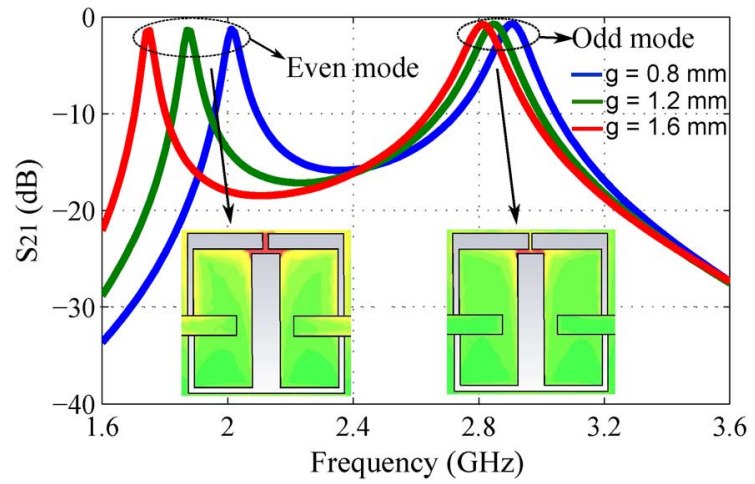

Fig. 3. Microstrip excited DMCSRR for studying the dependence of modal resonance frequencies on $g$ and the current distributions of the even and oddmode resonances. The geometrical parameters are: $w=0.3 \mathrm{~mm}, d=1.4 \mathrm{~mm}$ $l_{1}=8 \mathrm{~mm}, l_{2}=7.7 \mathrm{~mm}, l_{3}=6.9 \mathrm{~mm}, W_{f}=1 \mathrm{~mm}, g_{c}=0.2 \mathrm{~mm}$ and $g_{f}=1.6 \mathrm{~mm}$.

point of view, this is because there is no current flowing between the capacitive plates and ground plane through $L_{P}$ in the odd mode. Thus, the even and odd mode resonance frequencies can be calculated as

$$
\begin{aligned}
f_{\text {even }} & =\frac{1}{2 \pi \sqrt{\left(2 L_{P}+L_{S}\right) C_{C}}} \\
f_{\text {odd }} & =\frac{1}{2 \pi \sqrt{L_{S}\left(C_{C}+2 C_{M}\right)}} .
\end{aligned}
$$

As an illustration of the dual-mode behavior, a microstrip excited dual-mode CSRR is simulated by using the substrate with a relative dielectric constant of 10.7 and a thickness of $1.27 \mathrm{~mm}$. The length of the parallel inductive path $(g)$ is varied to demonstrate its effect on the even mode resonance. As shown in Fig. 3, the even mode resonance is directly dependent on $g$ whereas the odd mode is nearly unchanged. The small variation in the odd mode resonance is due to a small change in the capacitance $C_{C}$ as a result of varying the $g$. The current distributions for the two modes are shown in the insets of Fig. 3. For the even mode, the current flows through both $L_{P}$ and $L_{S}$ from the capacitive plates directly to the ground plane, whereas for the odd mode the current flows between the two capacitive plates through $L_{S}$ inductors.

\section{B. C-Shaped Coupling}

The $C$-shaped couplings have been chosen for the input/output feeds as shown in Fig. 4(a) to improve the

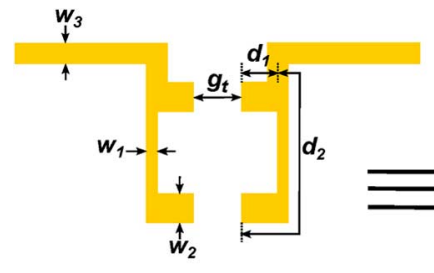

(a)

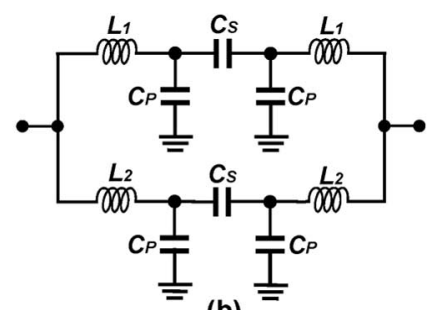

(b)
Fig. 4. $C$-shaped input/output feed (a) and its equivalent circuit model (b)

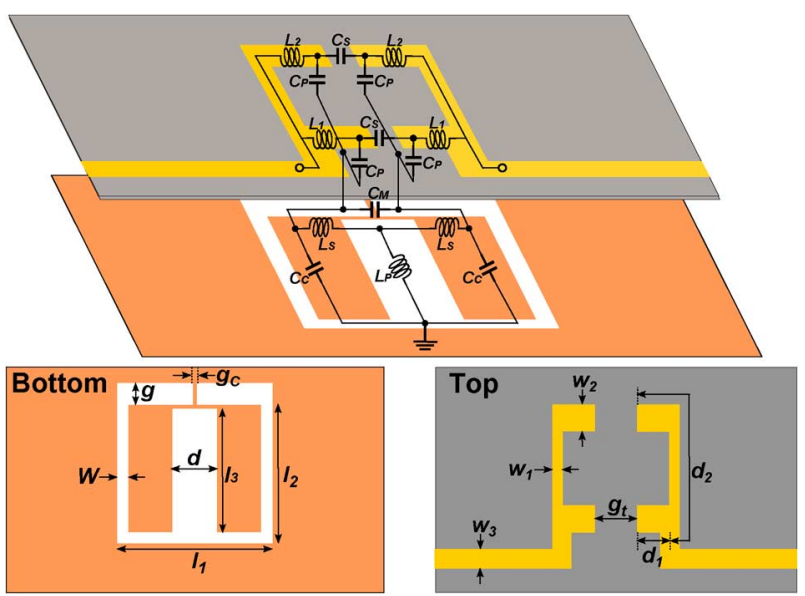

Fig. 5. Topology and equivalent circuit model of the proposed dual-mode filter. Orange layer shows the ground plane, microstrip metalization is yellow and the dielectric substrate is gray with thickness of $1.27 \mathrm{~mm}$ and $\epsilon_{r}=10.7$. The geometrical parameters are: $l_{1}=8 \mathrm{~mm}, l_{2}=7.4 \mathrm{~mm}, l_{3}=6.9 \mathrm{~mm}, w=$ $0.3 \mathrm{~mm}, d=1.4 \mathrm{~mm}, g=0.8 \mathrm{~mm}, g_{c}=0.2 \mathrm{~mm}, w_{1}=0.5 \mathrm{~mm}$, $w_{2}=1.5 \mathrm{~mm}, w_{3}=1 \mathrm{~mm}, d_{1}=3 \mathrm{~mm}, d_{2}=7.1 \mathrm{~mm}$ and $g_{t}=1.4 \mathrm{~mm}$. The electrical parameters are: $L_{1}=0.61 \mathrm{nH}, L_{2}=1.15 \mathrm{nH}, L_{S}=1.1 \mathrm{nH}$, $L_{P}=1.2 \mathrm{nH}, C_{M}=20 \mathrm{fF}, C_{S}=17 \mathrm{fF}, C_{P}=0.7 \mathrm{pF}$ and $C_{C}=1.65 \mathrm{pF}$.

transmission response in the passband [6]. The equivalent $L C$ model is available for $C$-shaped coupling as shown in Fig. 4(b). In this model, the values of $L_{1}$ and $L_{2}$ inductors can be controlled by the lengths $d_{1}, d_{2}$ and widths $w_{1}, w_{2}$. Also, the ratio $L_{1} / L_{2}$ is related to the location of the input/output microstrip lines $d_{1} / d_{2}$. The gap between the two $C$-shaped couplings is represented by the capacitive $\pi$-model constructed from the series capacitors $C_{S}$ and the parallel capacitors $C_{P}$.

\section{Filter Topology AND Circuit Model}

The proposed dual-mode filter and its circuit model are shown in Fig. 5. The circuit model incorporates the coupling effect between the $C$-shaped feeding microstrip lines and the DMCSRR embedded in the ground plane. The lumped element values of the circuit model are initially estimated by using the method presented in [13], and then, more accurate values are obtained by fitting circuit simulation results to electromagnetic simulation results.

Since the filter performance is dependent on its dimensions and geometry, the proposed filter and its circuit model are simulated in Advanced Design System (ADS) to observe the effect of different parameters. The substrate used for design and simulations is RT/duroid 6010 with a relative dielectric constant of $\epsilon_{r}=10.7$ and a thickness of $1.27 \mathrm{~mm}$. The filter bandwidth is mainly determined by the difference between the even and odd-mode resonance frequencies of the DMCSRR. 

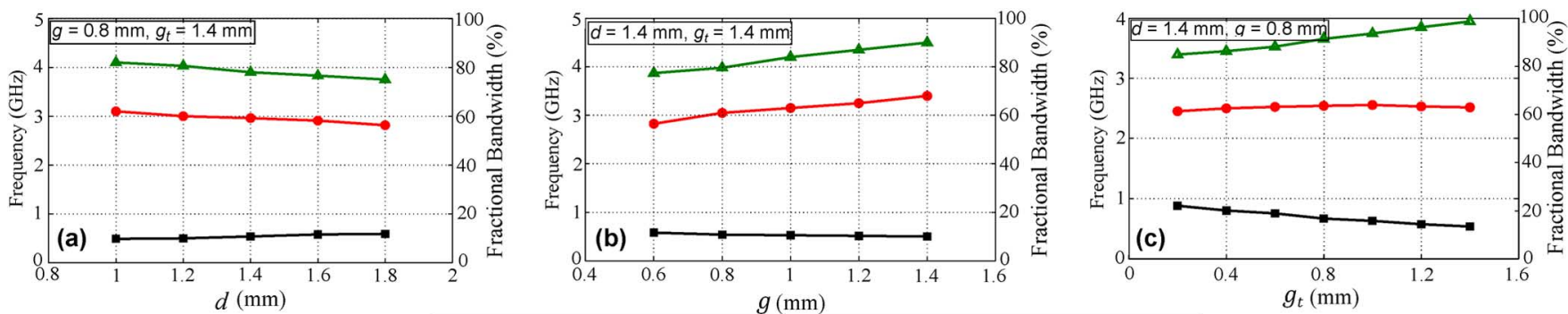

-Lower Zero Frequency $\rightarrow$ Upper Zero Frequency $\rightarrow$-Fractional Bandwidth

Fig. 6. Simulation results of parameter variation affect on fractional bandwidth and transmission zeros: (a) Filter performance versus $d$. (b) Performance versus g. (c) Performance versus $g_{t}$.

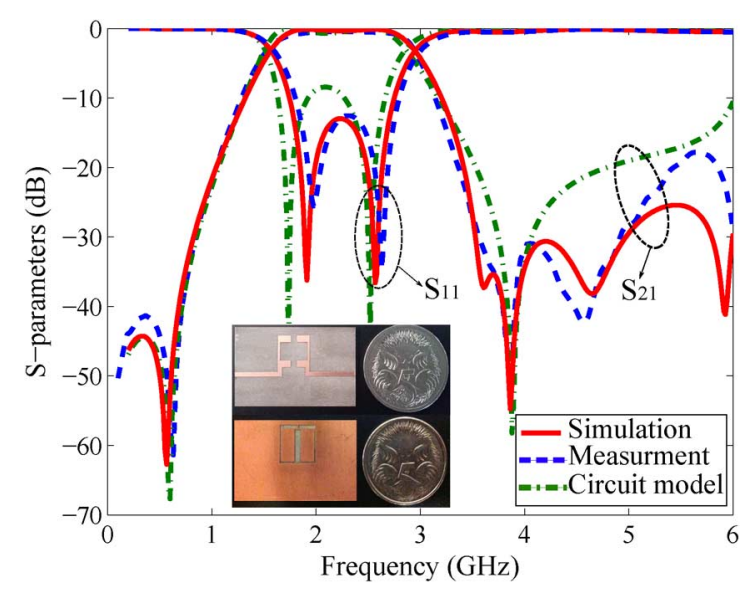

Fig. 7. Frequency response of the designed filter.

From (1) and (2), the even and odd-mode resonance frequencies of the filter can be tuned separately by using $C_{M}$ and $L_{P}$ or equivalently by changing $d$ and $g$ respectively. Increasing $d$ causes a smaller $C_{M}$ and a larger $L_{S}$, but $C_{M}$ is negligible for large enough $d$. Therefore, in this case, $L_{S}$ is dominant and the odd-mode resonance frequency will move downward decreasing the fractional bandwidth. A larger $g$ shifts down the even-mode resonance, which in turn causes a larger fractional bandwidth. The simulation results of varying $d$ and $g$ are shown in Fig. 6(a), (b), confirming the above explanation.

From the circuit model shown in Fig. 5, the transmission zeros of $S_{21}$ can be controlled through $C_{S}$ and $C_{P}$. These capacitances are dependent on the gap between the two $C$-shaped feeds [13]. As a demonstration, the frequencies of the upper and lower transmission zeros are determined by using electromagnetic simulation of the whole structure for different $C$-shaped coupling gaps. As shown in Fig. 6(c), by decreasing the $g_{t}$, the upper transmission zero frequency moves downward and the lower transmission zero moves upward.

\section{EXPERIMENTAL RESULTS}

A prototype of the proposed filter has been fabricated with the dimensions given in Fig. 5. The fabricated filter is depicted in Fig. 7. The simulated, measured and circuit model responses of the filter are shown in Fig. 7 over the 0.2 to $6 \mathrm{GHz}$ band. To avoid unexpected coupling of DMCSRR to the surroundings, the measurement is carried out on a non-metallic surface. As seen, the measurement are in a good agreement with simulations. The measured center frequency of the filter is $2.23 \mathrm{GHz}$ and the $-3 \mathrm{~dB}$ band-width is $1.38 \mathrm{GHz}$, which corresponds to a wide fractional bandwidth of $62 \%$. The maximum passband IL is $0.27 \mathrm{~dB}$ and the stopband is extended to $2.6 f_{0}$ below $-26 \mathrm{~dB}$; the maximum rejection is around $57 \mathrm{~dB}$, implying a good performance in suppressing unwanted out of band signals.

\section{CONCLUSION}

A wideband filter based on a DMCSRR has been presented and validated. An accurate circuit model is proposed for studying the effects of different parameters on the filter performance. The fabricated sample occupies a very compact area of $0.0625 \lambda_{g} \times 0.18 \lambda_{g}$ where $\lambda_{g}$ is the midband wavelength.

\section{REFERENCES}

[1] A. Horestani, C. Fumeaux, S. Al-Sarawi, and D. Abbott, "Split ring resonators with tapered strip width for wider bandwidth and enhanced resonance," IEEE Microw. Wireless Compon. Lett., vol. 22, no. 9, pp. 450-452, Sep. 2012.

[2] M. Gil, J. Bonache, J. Selga, J. García-García, and F. Martín, "Broadband resonant-type metamaterial transmission lines," IEEE Microw. Wireless Compon. Lett., vol. 17, no. 2, pp. 97-99, Feb. 2007.

[3] W. Withayachumnankul, K. Jaruwongrungsee, A. Tuantranont, C. Fumeaux, and D. Abbott, "Metamaterial-based microfluidic sensor for dielectric characterization," Sensors Actuators A: Physical, vol. 189, pp. 233-237, 2013.

[4] J. Naqui, M. Durán-Sindreu, and F. Martín, “Alignment and position sensors based on split ring resonators," Sensors, vol. 12, no. 9, pp. 11 790-11 797, 2012.

[5] I. A. Al-Naib, C. Jansen, and M. Koch, "Single metal layer CPW metamaterial bandpass filter," Progress Electromag. Res. Lett., vol. 17, pp. 153-161, 2010.

[6] J. C. Liu, D.-S. Shu, B. H. Zeng, and D.-C. Chang, "Improved equivalent circuits for complementary split-ring resonator-based high-pass filter with c-shaped couplings," IET Microw. Antennas Propag., vol. 2, no. 6, pp. 622-626, 2008.

[7] P. Mondal, M. Mandal, A. Chaktabarty, and S. Sanyal, "Compact bandpass filters with wide controllable fractional bandwidth," IEEE Microw. Wireless Compon. Lett., vol. 16, no. 10, pp. 540-542, Oct. 2006.

[8] M. Gil, J. Bonache, J. García-García, J. Martel, and F. Martín, “Composite right/left-handed metamaterial transmission lines based on complementary split-rings resonators and their applications to very wideband and compact filter design," IEEE Trans. Microw. Theory Tech., vol. 55, no. 6, pp. 1296-1304, Jun. 2007.

[9] X. Luo, H. Qian, J.-G. Ma, and E. Li, "Wideband bandpass filter with excellent selectivity using new CSRR-based resonator," Electron. Lett., vol. 46, no. 20, pp. 1390-1391, 2010.

[10] J.-C. Liu, H.-C. Lin, B.-H. Zeng, K.-D. Yeh, and D.-C. Chang, "An improved equivalent circuit model for CSRR-based bandpass filter design with even and odd modes," IEEE Microw. Wireless Compon. Lett., vol. 20, no. 4, pp. 193-195, Apr. 2010.

[11] L. Athukorala and D. Budimir, "Compact dual-mode open loop microstrip resonators and filters," IEEE Mirow. Wireless Compon. Lett., vol. 19 , no. 11, pp. 698-700, Nov. 2009.

[12] J. Baena, J. Bonache, F. Martín, R. Sillero, F. Falcone, T. Lopetegi, M. Laso, J. García-García, I. Gil, M. Portillo, and M. Sorolla, "Equivalentcircuit models for split-ring resonators and complementary split-ring resonators coupled to planar transmission lines," IEEE Trans. Microw. Theory Tech., vol. 53, no. 4, pp. 1451-1461, Apr. 2005.

[13] J. Bonache, M. Gil, I. Gil, J. García-García, and F. Martín, "On the electrical characteristics of complementary metamaterial resonators," IEEE Microw. Wireless Compon. Lett., vol. 16, no. 10, pp. 543-545, Oct. 2006. 